\title{
Occlusion-aware 3D Morphable Face Models
}

Bernhard Egger

bernhard.egger@unibas.ch

Andreas Schneider

andreas.schneider@unibas.ch

Clemens Blumer

clemens.blumer@unibas.ch

Andreas Morel-Forster

andreas.forster@unibas.ch

Sandro Schönborn

sandro.schoenborn@unibas.ch

Thomas Vetter

thomas.vetter@unibas.ch
Department of Mathematics and Computer Science

University of Basel

Basel Switzerland

http://gravis.cs.unibas.ch
We propose a probabilistic occlusion-aware extension to 3D Morphable Face Models [1, 2] for face image analysis based on the Analysis-bySynthesis setup. In natural images, parts of the face are often occluded by a variety of objects. Such occlusions are a challenge for face model adaptation. We propose to segment the image into face and non-face regions and model them separately. The segmentation and the face model parameters are not known in advance and have to be adapted to the target image. A good segmentation is necessary to obtain a good face model fit and vice-versa. Therefore, face model adaptation and segmentation are solved together using an EM-like procedure. We use a stochastic sampling strategy based on the Metropolis-Hastings algorithm for face model parameter adaptation [3] and a modified Chan-Vese segmentation for face region segmentation. Previous robust methods are limited to homogeneous, controlled illu- mination settings and tend to fail for important regions such as the eyes or mouth. We propose a RANSAC-based robust illumination estimation technique to handle complex illumination conditions. We do not use any manual annotation and the algorithm is not optimised to any specific kind of occlusion or database. We evaluate our method on a controlled and an "in the wild" database.

[1] Blanz and Vetter. A morphable model for the synthesis of 3d faces. In SIGGRAPH, 1999.

[2] Paysan et al. A 3d face model for pose and illumination invariant face recognition. In AVSS, 2009.

[3] Schönborn et al. A monte carlo strategy to integrate detection and model-based face analysis. In Pattern Recognition, 2013.

[4] Martinez and Benavente. The ar face database. In CVC Technical Report, 1998.

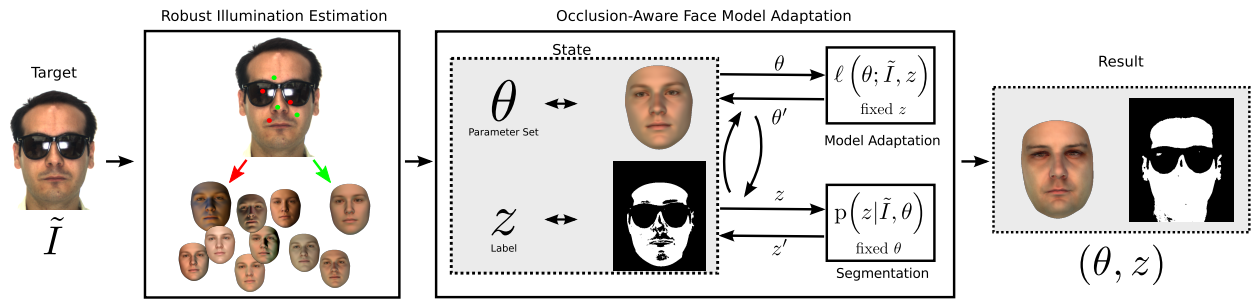

Figure 1: Algorithm overview: First we perform a RANSAC-like robust illumination estimation for initialisation of the segmentation label $z$ and the illumination setting. Then our face model and the segmentation are simultaneously adapted to the target image $\tilde{I}$. The result is a set of face model parameters $\theta$ and a segmentation into face and non-face regions. The presented target image is from the AR face database [4]. 